\title{
La auditoría financiera: Su importancia en las pequeñas y medianas empresas
}

\author{
Mónica Falconí- Hidalgo ${ }^{1}$ Silvia Altamirano-Bautista ${ }^{2}$ Nilda Avellán- Herrera ${ }^{3}$ Edison Cabezas-Mejía ${ }^{4}$
}

Fecha de recepción: 29 de Septiembre 2017

\section{Resumen}

Las pequeñas y medianas empresas (PYMES), por disposición de la Superintendencia de Compañías, Valores y Seguros del Ecuador (SC), a partir del 1 de enero de 2012 deben aplicar las Normas Internacionales de Información Financiera para Pequeñas y Medianas Empresas (NIIF para PYMES); éstas normas procuran se genere información financiera transparente y confiable tanto para clientes externos como internos; para lo cual se requiere información auditada que la certifique. La investigación tiene como finalidad revisar los principales referentes teóricos de la auditoría que permita a los lectores determinar su importancia en las PYMES, en este sentido se puede concluir que la auditoría permite evaluar el control interno, determinar debilidades y detectar fortalezas para evitar fraudes y corregir errores contables como duplicidad de asientos, mala asignación de cuentas, omisión de asientos, registro de valores incorrectos, entre otros.

Palabras clave: Auditoría financiera, información financiera, control interno, PYMES.

\section{Abstract}

Small and medium-size companies, by disposition of the Superintendency of Companies of Ecuador, as of January 1, 2012, must apply the International Financial Reporting Standards for Small and Medium-Sized Enterprises (IFRS for SMEs); these standards seek to generate transparent and reliable financial information for both external and internal clients; for which audited information is required to certify it. The research aims to review the main theoretical references of the audit that allows readers to determine its importance in SMEs, in this sense it can be concluded that the audit allows evaluating internal control, determine weaknesses and detect strengths to prevent fraud and correct accounting errors such as duplication of entries, misallocation of accounts, omission of entries, registration of incorrect values, among others.

Keywords: Financial audit, financial information, internal control, SMEs.

\section{Introducción}

El conocimiento de las bases conceptuales de la auditoría financiera permite determinar la importancia de este examen objetivo, sistemáti$\mathrm{co}$, profesional y posterior a las operaciones financieras en las empresas, para así dictaminar una opinión si están o no de acuerdo con las normas de contabilidad y los criterios financieros. El objetivo de esta investigación bibliográfica es revisar los principales referentes teóricos de la auditoría que permitan a los lectores determinar la importancia de este examen en las PYMES, ya sea por práctica sana o por disposición de la SC.

Una de las funciones de la auditoría es sentar las bases teóricas para obtener información financiera razonable y que el sistema de control interno integre mecanismos propios de verificación y evaluación que garantice el cumplimiento de los objetivos: salvaguardar los recursos, contar con información financiera confiable y suficiente, garantizar efectividad y eficiencia en las operaciones, y fomentar el cumplimiento de las leyes y regulaciones. En el desarrollo de la auditoría el auditor debe conocer las fases de auditoría como son diagnóstico preliminar, planificación, ejecución, comunicación de resultados. La utilidad de esta investigación es ampliar conocimientos en cuanto a los conceptos vinculados con la auditoría financiera, debido a la gran importancia que
Fecha de aceptación: 11 de Diciembre 2017

involucra la aplicación de una auditoría en las PYMES, así mismo se espera comprender y analizar la situación de este segmento de empresas, tomando en cuenta los cambios dispuestos por la SC acerca de la aplicación de la nueva normativa contable financiera. Es conveniente realizar la presente investigación para en un futuro estar preparados a los nuevos requerimientos que tienen las PYMES en lo que se refiere a auditoría y asesoría, además de desarrollar una investigación que sirva como referente teórico para futuras generaciones que se interesen por el tema.

\section{Metodología}

Para estudiar la importancia de la auditoría financiera aplicable a las PYMES, la metodología utilizada fue la investigación bibliográfica-documental, la misma que será considerada como parte esencial de un proceso de investigación científica. Así mismo este tipo de metodología permitirá indagar, interpretar, presentar datos e informaciones sobre el tema, utilizando para ello, una metódica de análisis con la finalidad de obtener resultados que pudiesen servir de base para futuras investigaciones.

La investigación permite cimentar las bases teóricas de la importancia de la auditoría financiera. De este modo se amplían las fronteras del conocimiento como un saber estratégico para el desarrollo de la profesión, y por esta vía, la construcción de una nueva sociedad, de que el contador debe apropiarse. En este orden de ideas se precisa la incorporación del método científico, permitiendo obtener nuevos conocimientos en el campo de la realidad social (Investigación pura)

\section{Resultados \\ Las Pequeñas y Medianas Empresas (PYMES)}

El término PYMES se usa para indicar o incluir entidades muy pequeñas sin considerar si publican estados financieros con propósito de información general para usuarios externos. En Ecuador para esta definición se consideran tres factores importantes como son ingresos totales, monto de capital y número de empleados; estos parámetros rigen para todas las empresas del sector ecuatoriano (García \& Villafuerte, 2015, p. 8).

Las PYMES se vuelven sistemas creadores de valor económico cuando desarrollan dinámicas productivas y competitivas que les permiten enriquecer su contexto, conectando a las personas con los mercados, en los cuales se encuentran los recursos requeridos para satisfacer sus necesidades, y a la vez se enriquecen en el proceso.

En este sentido, la búsqueda de competir en los mercados internacionales, la crisis económica, y la necesidad de sobrevivir ha llevado a buscar la reducción de costes, la optimización de procesos, la unificación de criterios. Éstas son necesidades imperantes en la PYME actual, que pueden solucionarse con información financiera confiable y veraz, en función de la aplicación de la normativa contable vigente y la validación de la misma a través de las auditorías financieras.

La fuerza competitiva de las PYMES ecuatorianas no solo está representada por la reducción del coste de mano de obra o de materiales, sino por la mejora continua de la información financiera que permite facilitar rapidez y flexibilidad en la evolución de los cambios. De acuerdo a datos publicados por la SC, de las 52.554 empresas que reportaron

\footnotetext{
Magíster en Auditoria Integral-Docente. Carrera Finanzas y Auditoría. Departamento Ciencias Económicas, Administrativas y de Comercio. Universidad de las Fuerzas Armadas-ESPE. SangolquiEcuador.E-Mail: mafalconi@espe.edu.ec

${ }^{2}$ Magíster en Tributación y Derecho Empresarial-Docente. Carrera Finanzas y Auditoría. Departamento Ciencias Económicas, Administrativas y de Comercio. Universidad de las Fuerzas Armadas-ESPE. Sangolquí-Ecuador. E-Mail: shaltamirano@espe.edu.ec

${ }^{3}$ Magíster en Gestión Financiera- Docente. Carrera Finanzas y Auditoría. Departamento Ciencias Económicas, Administrativas y de Comercio. Universidad de las Fuerzas Armadas-ESPE. San golquí- Ecuador. E-Mail: naavellan@espe.edu.ec

${ }^{4}$ Magíster en Docencia-Docente. Carrera Finanzas y Auditoría. Departamento Ciencias Económicas, Administrativas y de Comercio. Universidad de las Fuerzas Armadas-ESPE. Sangolquí-Ecuador. E-Mail: edcabezas@espe.edu.ec
} 
sus balances hasta el 30 de junio de 2016, 21.922 son PYMES y de este total, el $31 \%$ son consideradas medianas y el $69 \%$ pequeñas empresas.

Para tal efecto, la SC ejerce la vigilancia y control de las empresas en el orden jurídico, societario, económico financiero y contable, a más de proporcionar asesoramiento personalizado especialmente a las PYMES sobre diversos aspectos como actos jurídicos, constituciones, aumentos de capital, fusiones, transformaciones, escisiones, etc. La SC realiza una continua y cada vez más amplia segmentación de la información financiera, inversión societaria, inversión extranjera, entre otros aspectos, proveniente de cada una de las empresas bajo su vigilancia y control, a través de sistemas informáticos de tecnología avanzada, instrumentos que permiten disponer de información sólida y actualizada para diversos productos que desarrolla sobre la realidad nacional y la contribución de los diferentes segmentos empresariales al progreso socio económico del país.

Las PYMES reguladas por la SC obligatoriamente deben contar con información financiera de conformidad a las NIIF para PYMES, que les permitan tener información de calidad para tomar decisiones acertadas, misma que servirá de herramienta para ejecutar auditorías externas solicitadas por este organismo de control.

\section{Información financiera como base fundamental para la auditoría interna o externa en las PYMES}

"La información financiera es la que produce la contabilidad, indispensable para la administración y el desarrollo de las empresas, y por lo tanto, es procesada y concentrada para uso de la gerencia y personas que trabajan en la empresa" (Padilla \& Ediciones, 2012, p. 86). La información financiera es de gran importancia para comunicarse entre propietarios, directivos y organismos de control como usuarios de dicha información; además de que la publicación de la información financiera es una herramienta indispensable para las auditorías internas o externas.

Para la presentación de los informes contables se crearon un conjunto de normas internacionales, con el objetivo de estandarizar su presentación, tanto a nivel de grande empresa como para las PYMES:

Desde el año 2001 y hasta mayo de 2016, el IASB ha emitido 16 Normas Internacionales de Información Financiera (NIIF). Asimismo, fue promulgada la NIIF para Pequeñas y Medianas Entidades, que ya cuenta con dos versiones: 2009 y 2015. Por su parte, el órgano de interpretaciones correspondiente ha emitido los IFRIC (del inglés, International Financial Repoting Interpretation Committee) (Gómez Villegas, 2016, p. 16).

Las Normas Internacionales de Información Financiera para pequeñas y medianas empresas son una sola norma dividida en 35 secciones, su implementación es menos compleja que utilizar las NIIF completas: El Consejo de Normas Internacionales de Contabilidad, el 9 de julio de 2009, publicó las NIIF para pequeñas y medianas entidades (NIIF para PYMES), y desde entonces ha elaborado material de formación sobre la NIIF para PYMES, basado en sus 35 secciones mediante módulos de formación elaborados por el SMEIG (Martínez \& Ediciones, 2015, pp. 1-2).

En Ecuador a partir del 2009 se crea una tensión en los gerentes, contadores, y financieros con la disposición de la SC por la obligatoriedad de implementar las Normas Internacionales de Información Financiera y las Normas Internacionales de Contabilidad, al mismo tiempo esta disposición se constituyó en un reto y una oportunidad para los profesionales del área contable, lo que implicó lectura, capacitaciones y preparación para afrontar esta nueva normativa. Las compañías sujetas a la SC aplicaron el 1 de enero del 2012 las NIIF para PYMES siendo el período de transición el año 2011, existieron disposiciones por parte de la SC obligatorias para la implementación como: plan de capacitación, el respectivo plan de implementación, y fecha de diagnóstico de los principales impactos en la empresa, toda esta documentación debía ser enviada a la SC hasta el 31 de Octubre del 2011 aprobado por la junta general de socios o accionistas, o por el organismo que estatutaria- mente esté facultado para el efecto. Adicionalmente, estas empresas elaboraron, para sus respectivos periodos de transición lo siguiente: a. Conciliación del patrimonio neto reportado bajo NEC al patrimonio bajo NIIF al 1 de enero del 2011.

b. Conciliación del patrimonio neto reportado bajo NEC al patrimonio bajo NIIF al 31 de diciembre del 2011.

c. Conciliación del Estado de resultados del 2011, bajo NEC al estado de resultados bajo NIIF

d. Explicar cualquier ajuste material si lo hubiere al estado de flujos de efectivo del 2011 (Medina, 2012).

Las NIIF para PYMES que obligatoriamente se tuvieron que adoptar en el Ecuador en el año 2012, aseguran la calidad de la información toda vez que orientan el registro contable de la información de una empresa sin importar el carácter fiscal. La normativa faculta a las autoridades legislativas y reguladoras de cada país para que utilicen parámetros que permitan diferenciar PYMES, también establecen la facultad de determinar qué empresas están obligadas a aplicar dichas normas. Con la aplicación de estas normas se presentará un juego de estados financieros comparable a nivel mundial.

En este sentido, las empresas reguladas por la SC órgano regulador en Ecuador, ha dispuesto la obligatoriedad de las NIIF a todas las empresas y sociedades que están bajo su vigilancia y control, además de la obligatoriedad de aplicar auditorías externas que determinen el cumplimiento de éstas normas, como elemento clave en la toma de decisiones (Tene \& Castellanos, 2015, p. 282).

\section{Breve historia de la evolución de la Auditoría}

La auditoría en su concepción moderna tiene su origen en Inglaterra, según datos históricos se relaciona a fines del siglo XIII y principios del siglo XIV, en donde se auditaban las operaciones de algunas empresas privadas y las actividades de los funcionarios públicos que manejaban fondos del Estado; de ahí que la auditoría surge como consecuencia del desarrollo producido por la Revolución Industrial del siglo XIX. Los procesos de las empresas siempre se han evaluado enfocándose

Tabla 1. Breve historia de la evolución de la auditoría

\begin{tabular}{|c|c|}
\hline Años & Breve historia de la evolución de la Auditoria \\
\hline 1851 & Surge la primera asociación de auditores que se funda en Venecia \\
\hline 1862 & Se reconoce en Inglaterra la auditoria como profesión independiente \\
\hline 1917 & $\begin{array}{l}\text { El Instituto Americano de Contadores preparó a solicitud de la comisión federal } \\
\text { de comercio de USA la solicitud de auditorías al balance general. }\end{array}$ \\
\hline 1929 & $\begin{array}{l}\text { Se cambia de título a verificación de estados financieros, considerando la } \\
\text { importancia del estado de resultados. }\end{array}$ \\
\hline 1936 & $\begin{array}{l}\text { El Instituto Americano de Contadores emitió un folleto titulado examen de } \\
\text { estados financieros por Contadores Públicos Independientes, aparecieron dos } \\
\text { desarrollos de la profesión primero la verificación y segundo la responsabilidad }\end{array}$ \\
\hline
\end{tabular}

en la teoría de la contabilidad y el control, principalmente porque el control permite en una organización la evaluación del cumplimiento de los objetivos para obtener una información comparable con los mercados internacionales.

Las normas del International Accounting Standards Committee (IASC) constituyen un extenso y completo tratado de Contabilidad, mientras que, por su parte, la International Federation of Accountants (IFAC) ha conseguido un importante logro en pro de la armonización mundial del trabajo y condiciones personales de los expertos contables, mediante un amplio conjunto de normas que no solamente se ocupan de la auditoría sino también de los servicios relacionados con la misma, de la ética profesional, de la formación del auditor y de sus colaboradores, de la contabilidad y auditoría en el sector público y de la contabilidad de gestión internacional.

Sin duda los organismos internacionales como el Comité Internacional de Estándares de Contabilidad o la Federación Internacional de Contadores, han logrado estandarizar la información contable, así mismo la 
auditoría debe ser vista como un proceso que verifica todas las operaciones y actividades de una empresa garantizando una información confiable y la evaluación de control interno que permite salvaguardar los bienes, hacer fiable los registros y resultados de la contabilidad (Pereda, 2016, p. 72).

La falta de una información veraz y falta de ética de los profesionales han deteriorado la calidad de la información contable, al igual que el registro de operaciones ilícitas en diversas organizaciones, lo que exige se replantee y se adquiera responsabilidad en los procesos de control (Uribe, 2016, p. 170). Dentro de este marco, toda organización busca el éxito y eso se va a conseguir con información financiera veraz y relevante, que refleje la situación real de la empresa.

Al ser la auditoría un examen sistemático de los registros y las operaciones para determinar la razonabilidad, integridad, y veracidad de los saldos de los estados financieros la auditoría certifica que los sistemas de contabilidad de las empresas generen información confiable y verídica. La auditoría puede ser interna o externa, a continuación en la tabla 2 se presentan las diferencias entre éstos dos tipos de auditoría:

Tabla 2. Diferencias entre auditoría interna y externa

\begin{tabular}{|c|c|c|}
\hline & Auditoría externa & Auditoría interna \\
\hline Sujeto & Profesional independiente & Empleado \\
\hline Objetivo & Examen de los estados financieros & Control de operaciones y gestión \\
\hline Origen del encargo & Obligatoria, legal o estatutaria & Voluntaria \\
\hline Alcance & $\begin{array}{l}\text { Estados financieros en su conjunto } \\
\text { (Total) }\end{array}$ & $\begin{array}{l}\text { Restringida a áreas o procesos } \\
\text { concretos (parcial) }\end{array}$ \\
\hline Uso del informe & Empresa y público en general & $\begin{array}{l}\text { Restrin } \\
\text { da a la empresa / Institución pública }\end{array}$ \\
\hline Grado de independencia & Total & Limitada \\
\hline Responsabilidad & Profesional, civil y penal & Profesional \\
\hline Continuidad del trabajo & Periódico & Continuo \\
\hline Intensidad del trabajo & Menor & Mayor \\
\hline
\end{tabular}

Por disposición de la SC las empresas sujetas a su control deben realizar auditorías externas dependiendo del monto de sus activos, en este sentido son las grandes empresas las que generalmente implementan este tipo de control, sin embargo son muy pocas las PYMES que realizan auditorias continuamente, lo que les lleva a tener sistemas de control débiles dando lugar a fraudes, errores y perjuicios económicos. La mayoría de controles débiles radica porque las PYMES son familiares y su gobierno corporativo no ha diseñado procedimientos de control y seguridad para el manejo de los recursos, motivos suficientes para resaltar la importancia de la auditoría financiera que permita detectar éstas debilidades a tiempo y evitar perjuicios económicos futuros.

\section{La auditoría financiera y su importancia en las PYMES}

La auditoría financiera es un procedimiento que tiene por objeto revisar y comprobar, bajo un método especial, todas las cuentas y anexos en materia contable presentado en los estados financieros, para determinar si la información cumple con las normativas establecidas, con los objetivos y políticas fijadas en la empresa u organización, sugiriendo los cambios y mejoras que deban hacerse para el correcto cumplimiento de la gestión (Molina, Herrero, Pérez, \& Lara, 2014).

La auditoría financiera es el análisis de los estados financieros que tienen como finalidad determinar la razonabilidad de los saldos de la información económica-financiera utilizando afirmaciones como la veracidad, integridad, valuación y exposición (Enrique, Virginia, \& Ramón, 2014, p. 4).

En la elaboración de la auditoría financiera se utilizan las siguientes fases: diagnóstico general, planificación específica, ejecución, comunicación y seguimiento. El diagnóstico general consiste en el conocimiento de la organización, para tal efecto se diseña un cuestionario y una entrevista para disponer de instrumentos que permitan recaudar información del ente a ser auditado. Como productos de esta primera etapa se obtienen: los instrumentos diseñados y el conocimiento de la empresa. La etapa correspondiente a planificación permite la evaluación del control interno a través de cuestionarios, diagramas o descripciones permitiendo medir el riesgo y confianza. La ejecución, es el análisis de los datos e implica revisar las cuentas con sus auxiliares, mayores y registros, plasmando estos análisis en papeles de trabajo, los mismos que constituyen evidencia en caso de encontrarse hallazgos. En cuanto al informe de auditoría y las sugerencias, contiene el dictamen del auditor mediante su opinión, con sus conclusiones y recomendaciones.

Se determina que la importancia de la auditoría financiera en las PYMES en concordancia a la revisión bibliográfica realizada, es lograr una evaluación sincera de la situación financiera y administrativa, para obtener resultados confiables de sus actividades que les permitan, evitar errores de registro contable, verificar la efectividad del sistema de control interno, la aplicación correcta de los principios y la normativa tanto contable como tributaria, optimizar recursos así como mantenerse en el mercado, a través de las recomendaciones que pueda emitir el auditor externo en sus informes de auditoría. En la actualidad es indispensable contar con auditorías financieras por práctica sana, para determinar debilidades y corregir errores humanos que muchas veces se presentan en el desarrollo de las operaciones económicas, así como potencializar las fortalezas que se encuentran en el área contable.

Teniendo en cuenta la importancia de la Auditoría financiera, sobre el aseguramiento del debido proceso en cuanto a presentar información fiable para los tomadores de decisiones en función de informes de auditoría, que garanticen la razonabilidad de los datos presentados; en el Ecuador mediante resolución No. SCVS-INC-DNCDN-2016-011 de la SC emitida en septiembre 2016 se deroga la Resolución No. 02.Q.ICl.0012 del 11 de julio de 2002, referente a los montos mínimos de activos en los casos de auditoría externa obligatoria que eran de \$1.000.000 para las compañías nacionales anónimas, en comandita por acciones y de responsabilidad limitada; y, se establece como nuevo referente en su literal c lo siguiente: "Las compañías nacionales anónimas, en comandita por acciones y de responsabilidad limitada, cuyos montos de activos excedan los quinientos mil dólares de los Estados Unidos de América (US\$ 500.000,00)" están obligadas a someter sus estados financieros anuales al dictamen de auditoría externa (Superintendencia de Compañías Valores y Seguros, 2016, p. 3).

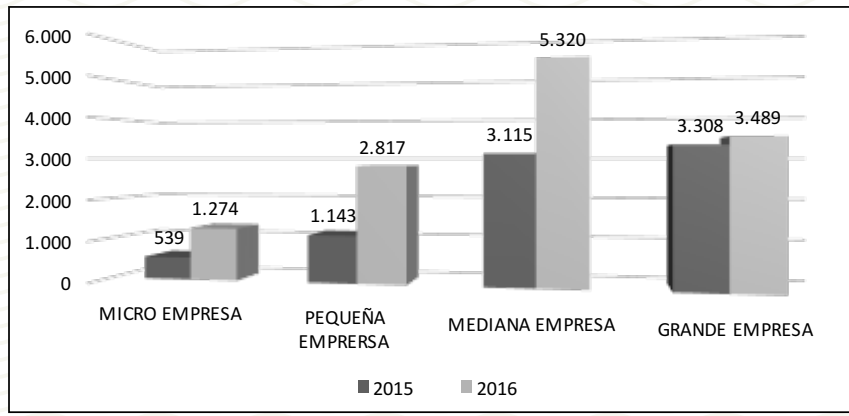

Fuente: Elaboración propia a partir de los datos publicados por la Superintendencia de Compañías Valores y Seguros (2017)

Figura 1. Número de empresas en Ecuador obligadas a presentar auditoría externa desde la publicación de la resolución No. SCVS-INC-DNCDN-2016-011 de la Superintendencia de

Compañías

Como se puede observar en la figura 1, por lo dispuesto en la resolución No. SCVS-INC-DNCDN-2016-011 de la SC, se refleja un incremento significativo en el volumen de empresas que deben contratar los servicios de una auditoría externa, resaltando principalmente el incremento del número de empresas en los segmentos de micro como de las pequeñas empresas. 


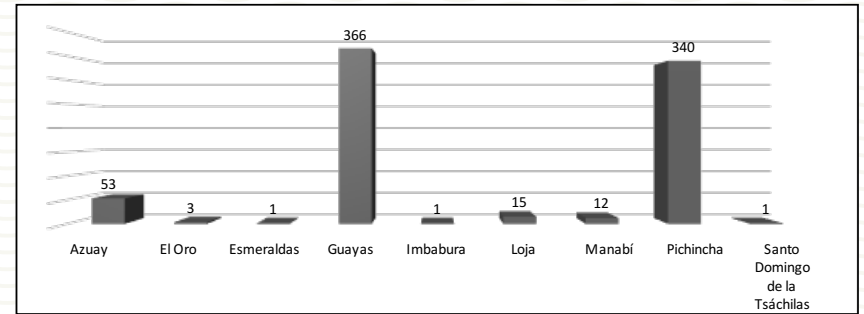

Fuente: Elaboración propia a partir de los datos publicados por la Superintendencia de Compañías Valores y Seguros (2017) Figura 2. Número de Personas naturales o jurídicas facultadas a realizar auditorías externas registradas en la Superintendencia de Compañías, Valores y Seguros del Ecuador

En relación a la figura 2, se destaca que de las 24 provincias del país, 10 son las que cuentan con personas naturales o jurídicas facultadas a realizar auditorías externas y que están debidamente registradas en la SC, sobresaliendo las provincias con mayor número de profesionales registrados Guayas, Pichincha y Azuay. Es necesario recalcar la valiosa labor que realizan los auditores en la emisión de los informes de auditoría, por tanto, habría que considerar que con el incremento del número de empresas que por exigencia de la entidad de control requieren de auditorías externas, se convierte en una oportunidad laboral para los auditores ya sean profesionales independientes o firmas auditoras.

\section{Reflexiones}

- Por las consideraciones anteriores, hay que resaltar la lucha continua de los empresarios por mantener sus emprendimientos, el esfuerzo y dedicación por conservar al grupo de trabajadores, cumplir con los pagos puntuales a proveedores, impuestos y contribuciones a los organismos de control, implementar reingenierías de procesos de producción, aplicar procedimientos de mejora continua, la contratación de auditorías externas que certifiquen su información financiera, todas éstas son inversiones que les permiten seguir en el mercado y poder ofrecer servicios y bienes de calidad a sus clientes para satisfacer sus necesidades.

- La auditoría debe suministrar información que debe encontrarse debidamente sustentada y servir de apoyo para los profesionales del área contable. En este sentido la SC ratifica la importancia de las auditorías, al considerar como requisito mínimo para presentar auditorías externas un monto de activos de $\$ 500.000$ (que anteriormente estuvo en $\$ 1.000 .000$ ) y lograr de esta manera que más empresas ejecuten este examen sobre la información presentada en los estados financieros. - La auditoría financiera permite que los evaluadores analicen la información financiera, evalúen el control interno, se determinen debilidades, detecten fortalezas, que permitan evitar fraudes y corregir errores, para un mejor desempeño empresarial así como parte de la ética y responsabilidad con los clientes externos como internos.

- El proceso de la auditoría en las pequeñas y medianas requiere la participación de todos los integrantes de la organización en la presentación de información y en la aplicación del plan de mejoras fortaleciendo la cultura del control.

- Las auditorías externas que exige la SC son una oportunidad laboral para los Auditores del país para cubrir la demanda existente, de las entidades que requieran de este servicio.

\section{Referencias}

Aumatell, C. S. (2012). Auditoría de la información : identificar y explotar la información en las organizaciones: Editorial UOC, S.L.

Cervantes, S., \& Yaima, K. (2014). Auditoría Especial al Subsistema de Nóminas de la Empresa Pecuaria Cuenca Lechera, Las Tunas.

Enrique, C. R., Virginia, B. V., \& Ramón, G. G. J. (2014). Análisis de estados financieros individuales y consolidados: Editorial UNED.

García, G. H., \& Villafuerte, M. F. (2015). Las restricciones al financiamiento de las PYMES del Ecuador y su incidencia en la política de inversiones. Actualidad Contable FACES, 18(30).

Gómez Villegas, M. (2016). NIIF y MiPYMES: los retos de la contabilidad para el contexto y la productividad (IFRS and SMEs: Accounting Challenges for Context and Productivity).

Martínez, Á. M. F., \& Ediciones, E. (2015). Contabilidad general con enfoque NIIF para las PYMES: Ecoe Ediciones.

Medina, G. R. (2012). Diccionario Práctico Tributario, Laboral y Societario. Ambato: Pedagógica Freire.

Molina, G. J. S., Herrero, I. S. M., Pérez, M. O., \& Lara, L. C. (2014). Fundamentos teóricos de auditoría financiera: Ediciones Pirámide. Ediciones.

Padilla, M. C., \& Ediciones, E. (2012). Gestión financiera: Ecoe

Pereda, J. T. (2016). Globalización y regulación contable. Algunos retos para nuestra profesión. Contaduría Universidad de Antioquia(32), 55-120.

Superintendencia de Compañías Valores y Seguros. (2016). Resolución No. SCVS-INC-DNCDN-2016-011. 19.

Superintendencia de Compañías Valores y Seguros. (2017). Ranking Empresarial 2017, from http://appscvs.supercias.gob.ec/ rankingCias/

Tene, J. M., \& Castellanos, J. Q. (2015). Papel de la Auditoria de Estados Financieros en las PYMES. Revista Publicando, 2(5), 277284.

Uribe, R. O. (2016). La información y el control en el proceso contable. Contaduría Universidad de Antioquia(42), 159-171. 